\title{
Site Selection and Deployment Scenarios for Servicing of Deep-Space Observatories
}

\author{
Harvey J. Willenberg, Michael A. Fruhwirth, and Seth D. Potter \\ Boeing Phantom Works \\ 5301 Bolsa Avenue \\ Huntington Beach, CA 92647-2099 \\ 714-372-9409 \\ harvey.j.willenberg@boeing.com \\ michael.a.fruhwirth@boeing.con \\ seth.d.potter@boeing.com \\ Stephen J. Leete and Rud V. Moe \\ NASA Goddard Space Flight Center \\ Greenbelt, MD 20771 \\ 301-286-9093 \\ stephen.leete@gsfc,nasa.gov \\ rud.moe@gsfc.nasa.gov
}

Abstract-- The deep-space environment and relative transportation accessibility of the Weak Stability Boundary (WSB) region connecting the Earth-Moon and Sun-Earth libration points makes the Sun-Earth L2 an attractive operating location for future observatories. A summary is presented of key characteristics of future observatories designed to operate in this region. The ability to service observatories that operate within the region around the Lagrange points may greatly enhance their reliability, lifetime, and scientific return. The range of servicing missions might begin with initial deployment, assembly, test, and checkout. Post-assembly servicing missions might also include maintenance and repair, critical fluids resupply, and instrument upgrades. We define the range of servicing missions that can be performed with extravehicular activity, with teleoperated robots, and with autonomous robots. We then describe deployment scenarios that affect payload design. A trade study is summarized of the benefits and risks of alternative servicing sites, including at the International Space Station, at other low-Earth-orbit locations, at the Earth-Moon L1 location, and on-site at the Sun-Earth L2 location. Required technology trades and development issues for observatory servicing at each site, and with each level of autonomy, are summarized.

\section{INTRODUCTION}

The NASA space science objectives for the coming decades include very ambitious goals. The science requirements for these missions will drive them to large collecting areas, multiple satellites in constellations to form sparsely filled apertures or separate focusing optics and focal planes. There is the possibility that these missions can be accomplished using "faster, better, cheaper" methods and by replacing entire spacecraft in case of significant failure or obsolescence. However, there are also missions with such large structures that they will likely require on-orbit assembly. Missions have such long life-time goals that failures will be likely that would limit life, and technology will advance so far over the desired life that the systems will become badly out of date. Many observing facilities are also of such complexity and high performance, and are so reliant on the space environment for their proper operation, that they will be difficult to fully verify prior to launch and will be likely to have subtle design flaws that will only be revealed on-orbit. The obsolescence of some of the original HST components was exacerbated by the lengthy launch delays related to the Challenger disaster, but at the current rate of progress computers and detectors become obsolete quickly.

The low Earth orbit (LEO) experience has shown that degradations in power available, complement of sensors remaining operational, sensor calibration \& sensitivity (after long-term exposure to space environment, especially radiation, wear of moving parts, etc.), effective communications bandwidth and on-board storage, can reduce an on-orbit asset to obsolescence and low value. An asset in space that has survived the launch environment, has its major systems operational and well-characterized, and has a limited set of well-understood problems, is a valuable space asset. Even if a servicer vehicle has mass comparable to a replacement vehicle, it may be re-used and amortized over several servicing missions and have higher reliability than a new scientific spacecraft.

Several servicing options are possible for these ambitious future missions. For a mission with one or more large space structures, the spacecraft may need assistance for assembly. If the structures are relatively rugged, they could be assembled in LEO, then pass through the Earth's radiation belts and out to their eventual on-orbit station. Other cases may require that assembly and servicing occur away from the various negative environmental factors characteristic of low Earth orbit.

Servicing in LEO from the Space Shuttle has been conducted on satellites which were not designed to be 
serviced on-orbit. All four were communication satellites, built by Hughes Aircraft Company, designed for operation in geostationary Earth orbit (GEO). In 1985 the Westar-IV and Palapa-B were retrieved and returned to Earth using the manned maneuvering system and the shuttle's Canadarm. They were both of the HS-376 dual-spinner type, and were still spinning when captured. In 1985 the Leasat-3 (or Syncom-IV) was captured, fitted with circuitry to bypass a balky switch, and retumed to service. In 1992, the Intelsat-6 satellite was retrieved with great difficulty, fitted with a new perigee kick motor, and returned to service. This was followed by the HST servicing missions in 1993, 1997, and 1999, with others planned for 2002 and 2004.

These and other missions demonstrated the capability and flexibility of humans in space using highly capable robotic tools (such as the Remote Manipulator System) and hand tools to capture and repair satellites with a wide range of repair needs. The ability to repair space-based telescopes through direct human intervention has tremendously expanded the scientific return of three telescopes on at least five different missions. The missions include those shown in Table 1 .

Table 1. Flight History of Space Telescope Servicing

\begin{tabular}{|l|l|l|}
\hline \multicolumn{1}{|c|}{ Vehicle } & Year(s) & \multicolumn{1}{c|}{ Activities, Accomplishments } \\
\hline $\begin{array}{l}\text { Space } \\
\text { Shuttle }\end{array}$ & 1984 & $\begin{array}{l}\text { Solar Max Mission recovery and repair, Palapa-B and WESTAR-IV recovery } \\
\text { and return to Earth. }\end{array}$ \\
\hline $\begin{array}{l}\text { Space } \\
\text { Shuttle }\end{array}$ & 1991 & $\begin{array}{l}\text { Contingency Gamma Ray Observatory antenna deployment, tested } \\
\text { extravehicular activity hardware }\end{array}$ \\
\hline $\begin{array}{l}\text { Space } \\
\text { Shuttle }\end{array}$ & 1993 & $\begin{array}{l}\text { Service HST (SM1): replace solar arrays, install WF/PC-2 \& COSTAR with } \\
\text { optical prescription corrections, gyros, magnetometers, and a 386 co- } \\
\text { processor, reboost }\end{array}$ \\
\hline $\begin{array}{l}\text { Space } \\
\text { Shuttle }\end{array}$ & 1997 & $\begin{array}{l}\text { Service HST (SM2): install STIS and NICMOS axial instruments, replace } \\
\text { FGS, install solid state recorder, replace reaction wheel, replace data interface } \\
\text { unit, replace magnetometers, and unplanned installation of blanket patches }\end{array}$ \\
\hline $\begin{array}{l}\text { Space } \\
\text { Shuttle }\end{array}$ & 1999 & $\begin{array}{l}\text { Service HST (SM3A): replace gyros (3), computer, FGS, SSR/tape recorder, } \\
\text { S-band transmitter, and install new outer blanket layers (2) }\end{array}$ \\
\hline
\end{tabular}

The current design guidelines are based on the servicing tools available and demonstrated, which are the Canadarm and various extravehicular activity (EVA) tools. Servicing is intended to occur based at the US Space Shuttle or the International Space Station (ISS) in LEO. Interfaces are to be compatible with the Canadarm 1 or 2 , or the Special Purpose Dexterous Manipulator (SPDM) that will be a part of the ISS. Future design features on satellites will be developed in parallel with servicers. Decisions will need to be made as to what capabilities to place on the satellite and which on the servicer. Developing servicers and satellites which are compatible, cost-effective and mass-efficient will be a challenge for the coming decade.

Other challenges include the relationship between humans, tools, robots, and spacecraft as to their location and function during servicing. This will include decisions as to whether humans will be at the worksite and how directly they interact with the hardware being serviced, and how to move the hardware and humans to their orbital locations.

In this paper, we discuss the characteristics of various orbital locations under consideration for scientific satellite operation, assembly, and servicing. This will focus on orbits about certain Sun-Earth and Earth-Moon libration points. We discuss the observatories being proposed for operation at various orbits. We discuss the range of on-orbit servicing activities, and the modes in which they can be performed. We look in some depth at several deployment scenarios, and establish a reference scenario for more detailed analysis. We describe the process for designing a spacecraft for servicing, and the preparation for a servicing mission. We will conclude with a summary of technology development requirements.

\section{VALUE OF LAGRANGE POINT HALO ORBIT LOCATION}

Space telescopes are currently operating with excellent results in Earth orbit and in deep space. Operating in LEO has one major advantage and several drawbacks. The major advantage is that low Earth orbit with inclinations below 57 degrees is currently accessible to astronaut crews for servicing. Other advantages to LEO operations are easy access by Shuttle or expendable launch vehicles (ELVs) and radiation protection provided by Earth's magnetosphere. Disadvantages are that satellites are in Earth shadow almost half of each 90-minute orbit; there is significant visible and infrared albedo and radiofrequency noise from the Earth; there is atmospheric drag to large telescopes and an environment of atomic oxygen and orbital debris. The low orbit means that the view of any given target is obstructed about $50 \%$ of the time by the Earth, and the shadowing results in deep thermal cycling during each orbit. These impacts of LEO are eliminated by operating in deep space away from the Moon and the Earth. Deep-space operations allow much longer periods of uninterrupted observing and a more stable environment for solar radiation. 
The Lagrange points are ideal locations for deep-space observatories. They maintain a position close enough to Earth (about 1.5 million $\mathrm{km}$ ) to allow constant communications with reasonably short delay times (10 seconds round-trip). They are also far enough from Earth to allow virtually continuous observation and to avoid Earth's microwave emission, magnetic field influence, and the other negative influences of noise and debris. They provide a very stable thermal environment. Sun-Earth L2 also provides constant access to the deep-space thermal cold sink at $2.7 \mathrm{~K}$, which enables passive cryogenic cooling of optics and detectors. Sun-Earth L1 has similar characteristics, except for some thermal loading and field-of-view blockage from the Earth, in addition to being about 3 million $\mathrm{km}$ closer to the Sun, with $4 \%$ higher solar intensity and daylight Earth albedo. They require minimal fuel to get from Earth orbit to the operating location. The Solar and Heliospheric Observatory [1] (SOHO) and Advanced Composition Explorer [2] (ACE) spacecraft are currently operating in halo orbits about the Sun-Earth L1 location, Genesis is en route to the Sun-Earth LI, and the Microwave Anisotropy Probe [3] (MAP) is en route to the Sun-Earth L2 location at the time of this writing. Future observatories that require cryogenic cooling, particularly the Next Generation Space Telescope, will probably operate at L2.

For satellites or constellations that are intended for operation at Sun-Earth L2 (S-E L2), the solar/thermal environment at Earth-Moon L1 (E-M L1) provides a fair approximation. Assuming a halo orbit that has very low delta-velocity to the S-E L2 orbit, the principal parameters of interest for the three orbital locations are given in Table 2. The anti-sun thermal loading from the Earth and Moon is about 44 times higher for E-M Ll than for Sun-Earth Ll, and $10^{5}$ higher than for Sun-Earth L2. The equivalent thermal sink temperature is (in the worst case) 18 times higher for E-M L1 than S-E L2, and about 2.5 times higher than for S-E LI. Note that if the Earth is shaded while at
E-M L2 and only the Moon is on the anti-sun side, the effective sink temperature drops to $43 \mathrm{~K}$ with a thermal flux of $199 \mathrm{~mW} / \mathrm{m}^{2}$... The E-M Lagrange point which most closely approximates S-E L2 is E-M L3, during the new Moon portion of the month. As soon as an out-bound spacecraft can get its sun shade to block the Earth, Moon and sun, it is effectively in its on-station environment. How quickly this occurs depends on details of the trajectory not available at this writing. In comparison to the MAP mission, it will take longer to leave the thermal influence of the Earth-Moon system because the departure velocity will be lower. Being effectively 'on station' during most of the transit can significantly reduce the impact of bringing a spacecraft back to E-M LI for servicing.

The energy required to reach Sun-Earth L2 is essentially escape velocity, i.e. to go from a $200 \mathrm{~km}$ circular Earth orbit to $\mathrm{L} 2$ requires a $\Delta \mathrm{V}$ of $3,200 \mathrm{~m} / \mathrm{s}$. However, because of the 4-body Sun-Earth-Moon-telescope system and the dynamics of the Weak Stability Boundary (WSB), the $\Delta V$ required to transfer between Sun-Earth L2 and Earth-Moon LI is extremely small and, in fact, vanishing under the right conditions. [4] For reference in the remainder of this paper, we assume that the transit time from LEO to L2 is approximately 90 days, as it is for the MAP mission. The $\Delta \mathrm{V}$ required to travel between S-E L2 and E-M L1 is less than $50 \mathrm{~m} / \mathrm{s}$, with windows that occur approximately monthly and transit times of approximately 90 days. [5] It is worthwhile to consider that observation can continue during the transit between the Lagrange points.

The stable manifold connecting these two Lagrange points allows us to consider them as traffic nodes: the windows open up monthly, the transit time is on the order of several months, and the energy required is very low. This allows us to consider moving the observatory and robotic equipment

Table 2. Thermal Characteristics of Lagrange Point Orbits

\begin{tabular}{|l|c|c|c|}
\hline Parameter & S-E Ll & S-E L2 & E-M LI \\
\hline Diameter of halo orbit $\left(10^{3} \mathrm{~km}\right)$ & $660 \times 150$ & $660 \times 150$ & TBD (assume 0) \\
\hline Period of halo orbit $($ days $)$ & 178 & 178 & 15 or 30 \\
\hline Distance from Earth $\left(10^{3} \mathrm{~km}\right)$ & $1645(\mathrm{TBV})$ & 1645 & 327 \\
\hline Distance from Moon $\left(10^{3} \mathrm{~km}\right)$ & $1645+/-384$ & $1645+/-384$ & 58 \\
\hline Time delay to Earth $(\mathrm{sec})$ & 5.5 & 5.5 & 1.08 \\
\hline Angular Radius of Earth $(\mathrm{deg})$ & .22 & .22 & 1.12 \\
\hline Angular Radius of Moon $(\mathrm{deg})$ & $.049-.079$ & $.049-.079$ & 1.72 \\
\hline Earth IR Thermal Flux $\left(\mathrm{mW} / \mathrm{m}^{2}\right)$ & 3.49 & 0 & 91 \\
\hline Earth Albedo Thermal Flux $\left(\mathrm{mW}^{2} \mathrm{~m}^{2}\right)$ & 7.32 & 0 & 190 \\
\hline Moon IR Thermal Flux $\left(\mathrm{mW} / \mathrm{m}^{2}\right)$ & .089 to .23 & 0 & 110 \\
\hline Moon Albedo Thermal Flux $\left(\mathrm{mW} / \mathrm{m}^{2}\right)$ & .073 to .19 & 0 & 89 \\
\hline Total anti-Sun Thermal Flux $\left(\mathrm{mW} / \mathrm{m}^{2}\right)$ & 11.0 to 11.2 & 0.0046 & $480(199 \mathrm{moon}$ \\
& & & only) \\
\hline Equivalent Sink Temperature $(\mathrm{K})$ & 21 & 3 & $54(43$ moon only) \\
\hline
\end{tabular}

* Thermal Flux to anti-sun side of $\mathrm{s} / \mathrm{c}$ 
back and forth between two points within the manifold to perform assembly and servicing at the optimum location. Either location is just as readily accessible from Earth. Since human spaceflight is much more sensitive to flight duration, we can consider E-M Ll as a site for human servicing.

There are a number of other aspects that make this an attractive site. Every point on the Lunar surface is equally accessible from E-M Ll. There are no launch windows between this location and Earth, which improves safety for human crews: it makes emergency return much more accessible. As traffic builds up in deep space, and assuming that water and/or hydrogen is recoverable from the Lunar surface, this location might serve as a storage depot for hydrogen recovered from the Lunar surface. This could then become a gateway and a fueling station for deep space human and/or robotic missions. As traffic volume warrants a return on investment, this location can become a hub for commercial investment: commercial ventures would include fuel, assembly of vehicles/missions that require multiple launches, and servicing/repair/maintenance of scientific instruments in deep space. For these reasons, the Earth-Moon LI halo orbit has been identified in the NASA Exploration Team (NEXT) technology roadmap as an optimal location for a space servicing infrastructure. [6-7]

\section{KEY CHARACTERISTICS OF WSB}

\section{OBSERVATORIES}

The NASA Office of Space Science has identified its science goals and objectives in the NASA strategic plans. [8-9] Implementation of many of the space science goals involves observatories located in space for distortion-free observation outside Earth's atmosphere. Among the missions considered for Lagrange sites are several with ambitious conceptual mission designs involving very large apertures, formation-flying arrays, long mission operational lifetimes, and other complexities. Generally, the means of establishing and maintaining implementation of these missions has not yet been determined. For example, the conceptual approaches for implementing large aperture structures in space include remotely-commanded mechanical deployments, remote-commanded robotic assembly, autonomous robotic assembly, or human-assisted robotic assembly. Mission operational modes envisioned include modular upgrade of key technology components, and life extension by periodic module replacement and refueling. Such missions comprise the candidates for inspace assembly and servicing. Table 3 describes a sampling of such cases and a suggestion of their possible in-space servicing benefits.

\section{SERVICING TASK CLASSIFICATION}

To devise a servicing architecture for a deep-space observatory, it is helpful to identify the tasks required, so that approximate requirements can be defined. A typical task will involve transferring some mass (e.g., structural components, instrumentation modules, propellant) to the observatory at a given servicing frequency, and will utilize instructions transmitted from a distance, or derived in-situ. Therefore, for each task, payload mass requirements, frequency of service, and latency must be estimated. This will aid in the decision as to where a given task takes place (e.g., low Earth orbit, in-situ, or a dedicated construction and servicing region in the Earth-Moon Ll region), what level of autonomy to use, and what technology is needed. For the purpose of this discussion, "service" is defined as the full range of tasks needed to bring a space telescope on line and maintain it. Service can therefore be classified into the following tasks:

- Assembly: includes initial assembly and checkout, as well as other activities that have similar requirements, e.g., major overhaul;

- Orbit transfer: includes delivery to operational orbit, return to servicing location, and end-of-life disposal;

- Resupply: mainly refers to propellant, but can include other fluids, etc.;

- Inspection and diagnosis: determining configuration state, need for interventions, etc.;

- Maintenance and repair; includes replacement of modules, refurbishment of structure, etc.; repair can be planned or unplanned; and

- Special: includes activities that do not readily categorize as part of the above tasks, such as space debris control and emergency operations.

These tasks and subtasks are listed in Table 4, along with rough estimates of payload mass requirement, frequency of service, and latency requirement. The estimates are generally categorized as high, medium, or low, with high indicating the greatest demand on resources, and so on. For latency, the categories are strict, medium, and lax, with strict indicating the most demanding situation (i.e., little or no significant time delay is acceptable). The most demanding requirements are shown in bold font in the Table, and the least demanding in italics. This categorization can aid in devising the location and method of performing each task. For example, high frequency-ofservice tasks may best be done in-situ (rather than transporting the observatory back to, say, LEO) and with a high level of autonomy (rather than incurring the expense of transporting astronauts to the operational site). However, if the latency requirement is strict and highly autonomous robots are not available, it may be necessary to retum the telescope to LEO, where servicing can be done through teleoperation from ISS and/or by EVA. Alternatively, astronauts could be sent to the operational site, if space transportation costs are reasonable or if a human space infrastructure exists beyond LEO. Conversely, the choice of location may determine the level of autonomy. For example, for initial assembly and major overhaul at ISS, astronauts are available for EVA and low-latency teleoperation; therefore, autonomous robotics may not be necessary. Level of autonomy ranges from none (i.e., human 
Table 3. Candidate Programs for In-space Assembly and Servicing at Lagrange Points

\begin{tabular}{|c|c|c|c|c|c|}
\hline $\begin{array}{c}\text { Candidate } \\
\text { NASA Programs }\end{array}$ & $\begin{array}{l}\text { Proposed } \\
\text { Sites }\end{array}$ & $\begin{array}{l}\text { Structure } \\
\text { Size }\end{array}$ & $\begin{array}{c}\text { Observing } \\
\text { Wavelength } \\
\text { Regime }\end{array}$ & $\begin{array}{c}\text { Assembly } \\
\text { (Reliability } \\
\text { enhancing } \\
\text { or Enabling) }\end{array}$ & $\begin{array}{c}\text { Servicing } \\
\text { (Productivity } \\
\text { enhancing } \\
\text { or Extension) } \\
\end{array}$ \\
\hline \multicolumn{6}{|c|}{ Astronomical Search for Origins } \\
\hline $\begin{array}{c}\text { Terrestrial Planet } \\
\text { Finder }\end{array}$ & $\begin{array}{l}\text { S-E } \\
\text { L2 }\end{array}$ & $15 \mathrm{~m}$ & IR & $\begin{array}{l}\text { - Structure } \\
\text { assembly } \\
\text { - Reflector } \\
\text { deployment } \\
\text { - Alignment } \\
\end{array}$ & $\begin{array}{l}\text { - Maintenance } \\
\text { - Upgrade }\end{array}$ \\
\hline Life Finder & $\begin{array}{l}\text { S-E } \\
\text { L2 }\end{array}$ & $40 \mathrm{~m}$ & $\begin{array}{l}\text { IR, Visible, } \\
\text { UV }\end{array}$ & $\begin{array}{l}\text { - Structure } \\
\text { assembly } \\
\text { - Reflector } \\
\text { deployment } \\
\text { - Alignment } \\
\end{array}$ & $\begin{array}{l}\text { - Maintenance } \\
\text { - Upgrade }\end{array}$ \\
\hline Planet Imager & $\begin{array}{l}\text { S-E } \\
\text { L2 }\end{array}$ & $\begin{array}{c}\text { Not } \\
\text { specified }\end{array}$ & $\begin{array}{c}\text { Visible, IR, } \\
\text { UV }\end{array}$ & $\begin{array}{l}\text { - Structure } \\
\text { assembly } \\
\text { - Reflector } \\
\text { deployment } \\
\text { - Alignment } \\
\end{array}$ & $\begin{array}{l}\text { - Maintenance } \\
\text { - Upgrade }\end{array}$ \\
\hline \multicolumn{6}{|c|}{ Structure \& Evolution of the Universe } \\
\hline $\begin{array}{c}\text { Space } \\
\text { Interferometry } \\
\text { Mission } \\
\end{array}$ & $1 \mathrm{AU}$ & $10 \mathrm{~m}$ & Visible & $\begin{array}{l}\text { - Optical bench } \\
\text { assembly } \\
\text { - Alignment } \\
\end{array}$ & $\begin{array}{l}\text { - Maintenance } \\
\text { - Upgrade }\end{array}$ \\
\hline $\begin{array}{c}\text { Laser } \\
\text { Interferometer } \\
\text { Space Antenna }\end{array}$ & $1 \mathrm{AU}$ & & $\begin{array}{c}\text { Laser } \\
\text { Interferometry }\end{array}$ & & $\begin{array}{l}\text { - Maintenance } \\
\text { - Upgrade }\end{array}$ \\
\hline Constellation $\mathrm{X}$ & $\begin{array}{l}\text { S-E } \\
\text { L2 }\end{array}$ & $8-10 m$ & X-ray & $\begin{array}{l}\text { - Optical bench } \\
\text { assembly } \\
\text { - Alignment } \\
\end{array}$ & $\begin{array}{l}\text { - Maintenance } \\
\text { - Upgrade }\end{array}$ \\
\hline $\begin{array}{c}\text { Early Universe } \\
\text { Observer }\end{array}$ & $\begin{array}{l}\text { S-E } \\
\text { L2 }\end{array}$ & $300 \mathrm{~m}$ & $X$-ray & $\begin{array}{l}\text { - Robotic } \\
\text { assembly } \\
\text { - Alignment }\end{array}$ & $\begin{array}{l}\text { - Maintenance } \\
\text { - Upgrade }\end{array}$ \\
\hline $\begin{array}{l}\text { Submillimeter } \\
\text { Probe }\end{array}$ & $\begin{array}{l}\text { S-E } \\
\text { L2 }\end{array}$ & $30 \mathrm{~m}$ & $\begin{array}{l}\text { FIR - sub- } \\
\text { millimeter }\end{array}$ & $\begin{array}{l}\text { - Structure } \\
\text { assembly } \\
\text { - Sunshields } \\
\text { deployment }\end{array}$ & $\begin{array}{l}\text { - Maintenance } \\
\text { - Upgrade }\end{array}$ \\
\hline \multicolumn{6}{|c|}{ Sun-Earth Connection } \\
\hline $\begin{array}{l}\text { Solar Polar } \\
\text { Imager }\end{array}$ & $\begin{array}{l}.5 \mathrm{AU}, \text { high } \\
\text { inclination }\end{array}$ & $\begin{array}{c}100 \text { m solar } \\
\text { sail }\end{array}$ & & $\begin{array}{l}\text { - Structure } \\
\text { assembly } \\
\text { - Sail deployment } \\
\end{array}$ & \\
\hline $\begin{array}{l}\text { Space Weather } \\
\text { Sentinel }\end{array}$ & $\begin{array}{c}.95 \text { AU sub } \\
\text { Ll }\end{array}$ & $\begin{array}{c}100 \text { m solar } \\
\text { sail }\end{array}$ & & $\begin{array}{l}\text { - Structure } \\
\text { assembly } \\
\text { - Sail deployment }\end{array}$ & \\
\hline \multicolumn{6}{|l|}{ Earth Science } \\
\hline $\begin{array}{l}\text { Geosynchronous } \\
\text { SAR }\end{array}$ & GEO & $30 \mathrm{~m}$ & RF & $\begin{array}{l}\text { - Structure } \\
\text { assembly }\end{array}$ & \\
\hline $\begin{array}{l}\text { Geosynchronous } \\
\text { LIDAR }\end{array}$ & GEO & $100 \mathrm{~m}$ & Visible & $\begin{array}{l}\text { Structure } \\
\text { assembly }\end{array}$ & \\
\hline Various missions & $\mathrm{L} 1, \mathrm{~L} 2$ & $>2.5 \mathrm{~m}$ & $\begin{array}{l}\text { IR, Visible, } \\
\text { UV }\end{array}$ & $\begin{array}{l}\text { - Structure } \\
\text { assembly }\end{array}$ & $\begin{array}{l}\text { - Maintenance } \\
\text { - Upgrade }\end{array}$ \\
\hline
\end{tabular}

Table 4. Space Telescope Servicing Requirements. 


\begin{tabular}{|c|c|c|c|c|}
\hline Tasks & & Requirement & of Service & Requirement \\
\hline \multirow{3}{*}{ Assembly } & Initial assembly & High & Once & Strict \\
\hline & Checkout at assembly location & Low & Low & $\operatorname{Lax}$ \\
\hline & Major overhaul & High & Low & Strict \\
\hline \multirow{3}{*}{$\begin{array}{l}\text { Orbit } \\
\text { Transfer }\end{array}$} & Delivery to final orbit & High & Low & $\operatorname{Lax}$ \\
\hline & Retrieval / return to servicing location & High & Low & $\operatorname{Lax}$ \\
\hline & End of life disposal & High & Once & $\operatorname{Lax}$ \\
\hline \multirow{2}{*}{ Resupply } & Propellant & High & Medium & $\operatorname{Lax}$ \\
\hline & Other fluids & Low & Low & $\operatorname{Lax}$ \\
\hline \multirow{7}{*}{$\begin{array}{l}\text { Maintenance } \\
\text { and Repair }\end{array}$} & Module changeout/replacement & Low & Low & Strict \\
\hline & $\begin{array}{l}\text { Refurbishment / retrofit of structure, } \\
\text { mirror segments }\end{array}$ & High & Low & Strict \\
\hline & Modification & Medium & Low & Strict \\
\hline & Decontamination & None & Medium & Lax \\
\hline & Cleaning / resurfacing & Low/None & Medium & \\
\hline & Test / checkout / inspection & None & Medium & Lax \\
\hline & Unplanned repair & Unknown & Unknown & Strict? \\
\hline \multirow{2}{*}{ Special } & Space debris control & Low & Low & \\
\hline & Emergency operations & Unknown & Low & Strict \\
\hline
\end{tabular}

Bold font indicates highest demand on resources and technology, italics indicate lowest level of demand, and normal font indicates intermediate level of demand.

EVA) to fully autonomous unsupervised robotics, with teleoperation in between. Teleoperation can be performed either on-site or remotely. The former may typically involve assembly of a telescope attached to, or in the vicinity of, ISS, with astronauts teleoperating a robot from ISS. If the latter (remote teleoperation), latency may be an issue. If latency becomes too great for the operator to use force feedback as a guide, then the remote operation can be viewed as a series of detailed uploaded commands, rather than true teleoperation. For example, the Sun-Earth L2 region is 5 light-seconds from Earth. There will therefore be a 10-second delay between the time a command is sent and the time a response is received at the ground site. Remote operation under such a circumstance is perhaps best seen as a series of uploaded commands, albeit a very detailed series; however, this is a matter of definition. Teleoperation from the Earth to the Moon ( $\sim 1.3$ lightseconds away) using visual feedback is feasible, though difficult. Autonomy is likely to involve less supervision as latency increases, as there will be less opportunity for a ground-based operator to override the autonomous system in time to prevent a problem. Autonomy can be categorized as follows:

1.0 Human EVA

2.0 Teleoperation

2.1 On-site teleoperation

2.2 Remote teleoperation

3.0 Autonomy

3.1 Supervised Autonomy

3.1.1 Require ground approval before execution
3.1.2 Allow ample time for ground override before the onboard system automatically carries out a command

3.1.3 Run autonomously, sending commands to the ground for occasional verification

3.1.4 Fully automated operations, with ground analysis only when a problem occurs

3.2 Unsupervised Autonomy

Level 3.2 can best be interpreted as minimally supervised autonomy, because completely unsupervised autonomy is probably not feasible or desirable for future advanced assembly, checkout, and service. However, unsupervised self-deployment is the rule for current deployment systems, and is one of the common failure mechanisms for commercial and civil satellites and spacecraft.

\section{RELATIVE MERITS OF ALTERNATIVE SERVICING SITES}

The site for initial deployment is dependent, in part, by the level of autonomy that will be available. An observatory capable of unsupervised self-deployment may be deployed at the operational WSB location. This represents the simplest case of assembly and transportation, but may not be realizable in the near-term, and may be the least forgiving, allowing little or no contingency if any part of the system fails. Teleoperated assembly is problematic due to the 5 -second one-way travel time for radio signals. This time lag precludes tactile feedback. 
In the near-term, LEO may be considered for assembly. There are several advantages of LEO, particularly in the vicinity of, or attached to, ISS. Perhaps most significant is easy accessibility of astronauts for EVA. Launching human missions to LEO is far less costly than launching to WSB or other "deep space" locations, both in terms of transportation costs, and in terms of consumables. If a continuously occupied ISS is available, the need to launch dedicated assembly crews can be minimized, or even eliminated. Teleoperation, from the ground, from ISS, or from a nearby occupied vehicle, can be accomplished with minimal latency. Principal disadvantages of LEO are contamination, drag, and orbital debris. The contamination issue becomes particularly acute if the assembly sequence is designed to take maximum advantage of the EVA capability. The use of
ISS may cause other issues to emerge, such as the availability of docking berths, and pointing issues arising from the need to minimize drag while keeping optical surfaces out of direct sunlight, and avoiding shadowing the ISS's solar arrays.

It may therefore be desirable to assemble the observatory beyond LEO, but at a relatively stable location whose transportation costs both from Earth and to the WSB location are acceptable. The Earth-Moon $\mathrm{L} 1$ region may meet these needs. A "kit" containing the telescope parts can be sent via low-thrust trajectory to this region. An uninhabited crew habitat and construction crane can be sent

Table 5. Assembly Sequence for DART-Like Gossamer Space Telescope.

\begin{tabular}{|c|c|c|}
\hline $\begin{array}{c}\text { Mission } \\
\text { Elapsed } \\
\text { Time } \\
\text { (days) }\end{array}$ & $\begin{array}{c}\text { Delta- } \\
\text { Elapsed } \\
\text { Time } \\
\text { (days) }\end{array}$ & Event \\
\hline 0 & 0 & $\begin{array}{l}\text { Launch telescope kit to Earth-Moon Ll using low-thrust orbital } \\
\text { transfer. }\end{array}$ \\
\hline 30 & 30 & Kit arrives at $\mathrm{LI}$; telemetry verifies condition. \\
\hline 40 & 10 & Launch habitat and crane to LI using low-thrust orbital transfer vehicle. \\
\hline 70 & 30 & Habitat and crane arrive at $L l$; telemetry verifies condition. \\
\hline 80 & 10 & Launch crew to LI using high-thrust Apollo-like vehicle. \\
\hline 83 & 3 & Crew arrives; begins checkout of habitat. \\
\hline 85 & 2 & Checkout of habitat complete; crew moves in. \\
\hline 86 & 1 & Crew unpacks kit. \\
\hline 88 & 2 & Crew assembles struts. \\
\hline 91 & 3 & Crew assembles sun shield. \\
\hline 94 & 3 & Crew installs telescope instrumentation. \\
\hline 95 & 1 & Crew attaches propulsion module to telescope structure. \\
\hline 96 & 1 & Crew installs gossamer reflectors. \\
\hline 100 & 4 & Crew aligns telescope \\
\hline 107 & 7 & Contingency \\
\hline 108 & 1 & Initiate low-thrust transfer of telescope to Sun-Earth L2 \\
\hline 109 & 1 & Crew departs for Earth, leaving habitat and crane at $\mathrm{Ll}$. \\
\hline 112 & 3 & Crew arrives at Earth. \\
\hline 198 & 86 & Telescope arrives at Sun-Earth L2 region. \\
\hline 198 & 0 & Orbit adjustment maneuvers initiated \\
\hline 203 & 5 & Telescope sees first light. \\
\hline 1116 & 913 & Telescope undergoes minor servicing in-situ. \\
\hline 2029 & 913 & Telescope undergoes major overhaul at Earth-Moon L1 \\
\hline 2942 & 913 & Telescope undergoes minor servicing in-situ. \\
\hline 3855 & 913 & Telescope undergoes major overhaul at Earth-Moon L1 \\
\hline 4768 & 913 & Telescope undergoes minor servicing in-situ. \\
\hline 5681 & 913 & Telescope undergoes major overhaul at Earth-Moon L1 \\
\hline 6594 & 913 & Telescope undergoes minor servicing in-situ. \\
\hline 7507 & 913 & Telescope is decommissioned. \\
\hline
\end{tabular}

Assemble at Earth-Moon L1; Operate at Sun-Earth L2. 
in a separate low-thrust launch. A crew can then rendezvous with the habitat using a high-thrust Apollo-like vehicle.

A possible assembly sequence is shown in Table 5 as an example. The mission illustrated here baselines a telescope using lightweight gossamer optics, though a similar sequence could be applied to a telescope using a rigid reflector system. The Delta-Elapsed time shown in the table for each step is the time at which a given step is initiated, not how long it takes. The total (cumulative) Mission Elapsed Time is derived from adding up the Deltas. Thus, the step "Crew aligns telescope", is initiated 4 days after "Crew installs gossamer reflectors". This implies that it can take up to 4 days to install the reflectors. The installation of the reflectors is done as late as possible in the assembly sequence to minimize contamination. The assembly sequence begins on day 86 with the unpacking of the telescope kit and concludes on day 108 with initiation of transfer to Sun-Earth L2. This includes 7 days of contingency to allow for slippage in the schedule and/or waiting for launch windows. Thus, total crew time to assemble the telescope is nominally $108-86-7=15$ days. More time may be needed if the crew needs to rest between EVA's, although rotating EVA crews could be used. The 30-day transfer time to $\mathrm{L} / \mathrm{l}$ is an estimate based on solar thermal propulsion. The $86+3+1=90$-day transfer time from Earth-Moon L1 to Sun-Earth L2 is based on a figure for transport to Sun-Earth L1 (presumably from LEO or Earth's surface) from a paper on the three-body problem. [4] A Delta-t of 0 means that the event is initiated at the same time as the previous event, but that the two events are distinct enough to warrant mention as separate steps. After first light, servicing is assumed to take place every 2.5 years, with every other time being major servicing back at Earth-Moon L1. The telescope can either have its own propulsion module, or it can utilize a low-thrust space tug. The latter may pay if there is more than one deep-space observatory in the Lagrange regions. Operational lifetime of the telescope is assumed to be 20 years.

\section{DEPLOYMENT SCENARIOS}

Based on the servicing task classification and the above analysis of the benefits and risks of alternative servicing sites, we can generate a trade space of various deployment scenarios. Assuming that the observatory is assembled in LEO and operates at Sun-Earth L2, there is a wide-ranging trade space around which to identify the optimal locations for servicing activities and the optimal mix of human and robotic cooperation. We have chosen to proceed with the following assumptions:

- The observatory is packaged into a single launch vehicle for initial assembly in LEO.

- The launch vehicle payload includes a transfer stage to carry the assembled observatory to its operational orbit at Sun-Earth L2.

- Servicing will be performed at Earth-Moon L1.

With these assumptions, we have developed a concept of operations with the steps shown in Table 6. The table also lists options for key decisions.

Table 6. Concept of Operations

\begin{tabular}{|c|c|c|}
\hline $\begin{array}{l}\text { Step } \\
\text { No. }\end{array}$ & Description & Options \\
\hline \multirow{2}{*}{1} & \multirow{2}{*}{ Package observatory into launch vehicle } & $\alpha$. Expendable launch vehicle \\
\hline & & B. Space Shuttle \\
\hline \multirow[t]{2}{*}{2} & \multirow[t]{2}{*}{ Launch to an ISS orbit } & $\begin{array}{l}x . \text { The crew comes from ISS or a later Shuttle } \\
\text { flight }\end{array}$ \\
\hline & & y. The crew launches with the observatory \\
\hline \multirow{4}{*}{3} & \multirow{4}{*}{ Assemble the observatory in LEO } & A. Fully autonomous robotic assembly \\
\hline & & B. Automated assembly with crew backup \\
\hline & & C. Tele-operated assembly \\
\hline & & D. EVA assembly \\
\hline 4 & $\begin{array}{l}\text { Check out the observatory in LEO and } \\
\text { repair/adjust as required }\end{array}$ & \\
\hline 5 & $\begin{array}{l}\text { Transport the observatory from LEO to S-E } \\
\text { L2 }\end{array}$ & \\
\hline 6 & $\begin{array}{l}\text { Check out the observatory \& operate at S-E } \\
\text { L2 }\end{array}$ & \\
\hline 7 & $\begin{array}{l}\text { Transfer from S-E L2 to E-M L1 for } \\
\text { maintenance }\end{array}$ & \\
\hline \multirow{4}{*}{8} & \multirow{4}{*}{ Service the observatory } & A. Fully autonomous robotic servicing \\
\hline & & B. Automated servicing with crew backup \\
\hline & & C. Tele-operated servicing from Earth \\
\hline & & D. EVA servicing \\
\hline 9 & $\begin{array}{l}\text { Transport the observatory from E-M L1 to } \\
\text { S-E L2 }\end{array}$ & \\
\hline
\end{tabular}


Each of the options listed in Table 6 represents a branch in a decision matrix. In principal, the options in the third column represent a matrix of $2 \times 2 \times 4 \times 4=64$ combinations. Actually, some of these combinations can be eliminated without further study. For example, if option A were selected for step 8 (fully autonomous servicing at Earth-Moon L1), then we would assume fully autonomous servicing capability, and there would be no reason to transfer the observatory to the Earth-Moon system for servicing - it can be better performed on-site at S-E L2. On the other hand, option $\mathrm{C}$ has two variations for step 8: teleoperations can be performed with an Earthbound crew when the observatory is either at E-M L1 or at E-S L2, provided that a tele-operations arm has been placed at the servicing location with the necessary support equipment and utilities. Assuming the servicing is done at E-M Ll, or at any location outside LEO, option $B$ requires a human habitat nearby, presumably at $\mathrm{L} 1$. Option $\mathrm{D}$ requires both a habitat and an arm at the servicing site.

We will proceed with a specific reference architecture as a baseline around which to perform trade studies. The reference architecture emphasizes operations by human crew using extravehicular systems. The decision branch includes a concept of operations that we define here as $1 \beta$ $2 x-3 D-4-5-6-7-8 D-9$. This branch was selected to optimize the capability for the human crew to make unscheduled adjustments to the observatory, assuming that EVA repair is more cost-effective than either operating with a defective observatory or completely replacing the observatory. Trade studies around this reference architecture are planned.

\section{DESIGN GUIDELINES FOR SERVICING}

The range of robotic servicing capabilities under development is wide. The Orbital Express [10] is an example of a fully robotic, automatic, modest system. Under this model, a servicing spacecraft will perform a rendezvous and docking, adding itself to the original spacecraft. This additional equipment can include replacement, upgrade or even additional electronics units, propellant, and perhaps other resources. The host system must be capable of integrating the new components. This model might apply for servicing in situ at S-E L2, under the assumption that we have not yet attained the capability to send humans to S-E L2.

Using a different approach is the Robonaut currently under development at the Johnson Space Center. [11] This anthropomorphic, teleoperated robot is designed to use the set of tools already qualified for use in space by humans in spacesuits - this has intuitively obvious control modes such as immersive tele-presence tele-operation. While the effects of time-delay are still under study, it may become feasible to teleoperate Robonaut or similar robots at E-M L1 from Earth, accepting some reduction in time efficiency in trade for safety and cost. Additional applications are considered with the human operator in close proximity, but still safe from extreme or hazardous environments.

A major issue for future science missions, especially for those which include large, delicate structures and optics, is the possibility of damage of the mission hardware by the servicing agent. The approach being taken on the International Space Station is to ruggedize all hardware, with few exceptions, to be able to withstand a 125-lb kick load over a half-inch diameter area (4.4 MPa). Clearly no gossamer or optics structure would meet this, calling into question the feasibility of human servicing. At issue is both safety and mission success. It may be feasible to design missions that are mostly not hazardous under these loads but which do have many surfaces which could be damaged by a kick load. There is also concern that loads imparted to one portion of a spacecraft might be transmitted to a more delicate structure, as is the case for the flexible roll-up style solar arrays used on HST. It may be necessary to put substantial effort into preventing this type of damage, by a control system that reduced the loads transmitted into delicate structures to nearly zero. Note that for HST, the solution to this problem is to replace the original delicate arrays with robust, smaller and more efficient arrays. This is a design approach enabled by the lifting capability of the Space Shuttle that will generally not be available for missions to more exotic orbits due to propulsion and launch limitations.

In general, the current set of requirements and standards for satellite servicing are for the Space Shuttle and the International Space Station. It may not be feasible to design hardware to these standards to perform the ambitious science missions under consideration. Interaction is needed between the EVA and robotic servicing communities, spacecraft designers, and scientist customers to determine appropriate requirements for this new regime of space operations. Toward this end, efforts are underway by one of the authors (Leete) to update the AIAA Guide G-042, Design for On-Orbit Spacecraft Servicing, with the ultimate goal of establishing a design standard that is the result of this interaction.

The paradigm for robotic and human servicing has generally been to have unique interfaces. For Space Shuttle missions, robotic interfaces should have over-ride features that allow human servicers to take contingency actions in case the nominal operation fails. This can lead to a confusing mixture and duplication of interfaces. One of the attractive features of Robonaut is that it allows the designer to design to one set of interface standards, the one for human EVA operation. Then, the work can be done either by humans or Robonaut. If other robots can also be made to work with standard EVA interfaces, then a wider range of servicing options will be open. 
From the spacecraft designer perspective, the preference would be to not have to consider servicing. From an ease of servicing, close attention to standards and a robust design is preferred. Another area needing further work is a next generation of EVA interfaces that satisfy EVA requirements for ease and speed of service, yet add minimal weight to the system. Early EVAs established that a dense population of handrails on a worksite was extremely valuable to a spacewalker who is free-floating. The weight of these additions is significant, however. In the current time frame it is hard to imagine weight not being at a premium for objects being boosted to high, energetic orbits. However, if future capabilities advance to the point of sending servicing hardware to the same energy orbits and if servicing accommodation is as critical as it is now, perhaps we will put handrails all over the surface of a S-E L2 observatory. If the servicer can use dextrous manipulation to grasp and traverse a field of lightweight hard points, integrated into the structure and outfitted with targets and markings, perhaps the burden of servicing accommodation can be vastly reduced.

If starting from the preliminary design phase, here are a number of design steps related to servicing:

- Define S/C subsystems, subsystem components, requirements

- Define, group and select servicing events, define method of performing servicing

- Preventative/routine, scheduled, unscheduled, accidental

- Define serviceable modules (orbital replacement units /ORU's)

- Define servicing scenario (timing, sequence, parts production, mock-ups)

- Detailed design of $\mathrm{s} / \mathrm{c}$, ORUs

- Verify servicing by analysis, neutral buoyancy testing.

A fair amount has been written on how to perform servicing. Currently, this means working closely with the Space Shuttle Project Office (SSPO) at the Johnson Space Center. They require extensive documentation to cover what the servicing customer, such as the HST project at the Goddard Space Flight Center, needs. This includes Flight Rules, which detail the guidelines within the servicer can act, including thermal limitation, mechanical loads, minimum installation criteria, etc. The customer provides a complete description of his system. The customer provides computer models and physical models of his hardware, for crew training in underwater training, virtual reality training, etc. The customer provides a high fidelity mockup of the flight hardware already on orbit, as well as the flight hardware being installed as part of the servicing activity, for hands on "crew familiarization" training. The customer will also participate in the mission, and will be part of the ground operations team, and will therefore need to be trained for this via simulations or rehearsals before the mission. The customer and servicer will need to develop and practice the servicing procedures, as well as contingency procedures.
Early servicing missions at E-M Ll would probably be similar in terms of process. NASA likely would still be the servicing provider, and would build on its LEO experience base and require those products and participation from the customer that had proven vital in the past. When servicing is more mature, it would be a commercial operation, comparable to the current state of launch services. Preparations for servicing missions will typically have challenging elements due to the remoteness of the servicing location, the elements of zero-gravity and vacuum which are so difficult to fully simulate on Earth, and the difficulty of being sure that new components will fit onto the existing hardware without further modification.

Early servicing may be mostly robotic, perhaps using a robot that had proven itself on the ISS. Candidates for this are the Special Purpose Dextrous Manipulator (SPDM), which is based on the Flight Telerobotic Servicer of the early 1990's and made by MDRobotics; Robonaut, under development at the Johnson Space Center; and Ranger [12], currently a research project at the University of Maryland. These are all two-armed dextrous robots, intended to perform servicing activities on the ISS.

\section{TECHNOLOGY DEVELOPMENT}

\section{REQUIREMENTS}

A summary of the key technology development requirements for in-space assembly and servicing of deepspace observatories is shown in Table 7. These needs fall in five general categories: (1) large space structure assembly techniques; (2) improvements in EVA suits; (3) parts and processes standardization; (4) in-space transportation modeling and vehicle development; and (5) effective, interactive cooperation with astronauts and robots

Although a number of technology studies have been conducted to understand large space structures assembly techniques, most of these have focused on understanding of the thermal and mechanical dynamic stability of the structures. There have been several missions that have involved in-space repair and maintenance of telescopes and satellites, but the system which currently has used the most extensive tool kit of in-space assembly is the ISS. This is being assembled in low Earth orbit with a combination of EVA conducted by astronauts and telerobotic operations manipulated by astronauts aboard the ISS, Space Shuttle, and Soyuz. An objective survey of the positive and negative attributes of the ISS assembly operations would be a very useful step in developing guidelines for in-space assembly and servicing of large observatories. The processes for observatories are likely to be different than those for ISS, since the observatories will operate in locations other than LEO, with longer communications latency and fewer opportunities for transporting additional supplies and crew. Observatories will typically be much lighter, and more critical for precision than the ISS. 
Table 7. Technology Needs for Deep-Space Observatory Assembly and Servicing

\begin{tabular}{|c|c|c|}
\hline Technology Requirement & Applications & Current Technology Status \\
\hline Lagrange manifold models & $\begin{array}{l}\text { Trajectory planning for servicing } \\
\text { schedules }\end{array}$ & $\begin{array}{l}\text { JPL models of 4-body dynamics } \\
\text { (LTool) }\end{array}$ \\
\hline $\begin{array}{l}\text { LEO to } L I \text { transportation for } \\
\text { science facilities and resupply }\end{array}$ & $\begin{array}{l}\text { Delivery of observatory facility to } \\
\text { deep space } \\
\text { Delivery of resupply/repair } \\
\text { components, fuel, and robots to } \\
\text { L1 }\end{array}$ & $\begin{array}{l}\text { Electric thrusters for efficient } \\
\text { transition }\end{array}$ \\
\hline $\begin{array}{l}\text { LEO to } L I \text { transportation for } \\
\text { human crew }\end{array}$ & $\begin{array}{l}\text { Delivery of crew to service/ } \\
\text { repair observatory at } L \text { ] }\end{array}$ & $\begin{array}{l}\text { Space Shuttle (STS) and Space } \\
\text { Station (ISS) systems operating in } \\
\text { LEO } \\
\text { No current plans for human } \\
\text { vehicles beyond LEO } \\
\text { Transfer vehicle architecture } \\
\text { concept studies in work } \\
\end{array}$ \\
\hline $\begin{array}{l}\text { L1 facility for crew habitation, } \\
\text { assembly and servicing } \\
\text { operations }\end{array}$ & & $\begin{array}{l}\text { Human safety evaluations in } \\
\text { work } \\
\text { Facility architecture concepts } \\
\text { studies in work }\end{array}$ \\
\hline $\begin{array}{l}\text { Large space structures assembly } \\
\text { techniques }\end{array}$ & Initial assembly of observatory & $\begin{array}{l}\text { ISS construction (EVA \& } \\
\text { telerobotic assembly) } \\
\text { HST experience } \\
\text { Gossamer telescope assembly } \\
\text { concept studies in work } \\
\text { Rigid telescope assembly concept } \\
\text { formulation } \\
\text { Truss assembly techniques } \\
\text { demonstrated in neutral buoyancy }\end{array}$ \\
\hline $\begin{array}{l}\text { Large space optics mechanical } \\
\text { stability }\end{array}$ & $\begin{array}{l}\text { Alignment of assembled critical } \\
\text { structures }\end{array}$ & Concept formulation \\
\hline Parts and process standardization & $\begin{array}{l}\text { Simplify resupply inventory } \\
\text { Facilitate standard robotics } \\
\text { processes }\end{array}$ & $\begin{array}{l}\text { ISS \& STS manifesting, tools, \& } \\
\text { processes }\end{array}$ \\
\hline EVA suit & $\begin{array}{l}\text { Enhance human capabilities for } \\
\text { work duration, manipulation, } \\
\text { visibility, safety }\end{array}$ & Shuttle and Orlan suits \\
\hline $\begin{array}{l}\text { Human/robotic cooperative } \\
\text { operations techniques }\end{array}$ & $\begin{array}{l}\text { L1, L2 assembly, service \& repair } \\
\text { Planetary exploration } \\
\text { Enable scale extension and } \\
\text { complexity in assembly } \\
\text { Enhance science performance, } \\
\text { reliability, mission duration }\end{array}$ & $\begin{array}{l}\text { ISS assembly } \\
\text { HST servicing missions } \\
\text { Capabilities state of art } \\
\text { evaluations and projections in } \\
\text { work } \\
\text { Deep sea/oil/hazardous } \\
\text { environments }\end{array}$ \\
\hline
\end{tabular}

The complexity of robotic systems and processes is closely related to the number of different procedures required and the degree to which the components appear to be unique. Robotic systems can be made more autonomous if there are fewer unique parts and if the parts to be assembled have standard sizes, shapes, and markings to facilitate identification of parts, orientation, and function. Standardization would also greatly ease logistics, thus decreasing inventory while increasing the likelihood that a repair part is already in space when needed.

Human-robotic optimization can be expected to provide enhanced performance in-space assembly and servicing complex tasks, particularly where uncertainties are numerous, dynamic, not well structured, or difficult to model. The interplay of human consciousness with 
optimized robotic equipment will extend the capabilities for reliably deploying large, complex facilities and observatories. The particular roles for the human operators and controllers, the dynamic information requirements (including latency), the interaction with subtleties and unexpected events or results requiring anticipation or replanning, will determine whether the human presence is required in close proximity on-site or may more optimally be located remotely. Cost considerations of the required supporting safety and habitability accommodations should be compared in a variety of scenarios, searching for a breakover point where the productivity justifies the investments. This may be found where there are a large variety of missions to be addressed in the optimization, including for example, planetary explorations.

In order to assemble and service these large space structures with humans the EVA suit technology needs to advance. The locations where the assembly and servicing may take place will require suits that provide better protection against radiation and debris, allow longer EVA times, require less crew preparations and maintenance overhead and require less physical stamina. The nature of the structures involved in the construction of these structures demands a suit with significantly more dexterity, improved visibility and enhanced mobility and have less mass. Communications and in-suit data display and gathering systems are also areas that through improvements will provide the crew and the ground station the necessary tools to increase the productivity of EVAs.

The mathematical modeling of invariant manifolds and the weak stability boundary between the Earth-Moon and SunEarth Lagrange points is a very complex four-body problem, involving the Sun, Earth, Moon, and the observatory. Although it is understood that the $\Delta \mathrm{V}$ requirements for transfer between Earth-Moon L1 and Sun-Earth L2 are trivial, the level of understanding of the dynamics is not yet sufficient to address transit times, other than by individual trajectory analysis of selected opportunities. A deeper understanding of the time required to transfer between the Lagrange points would help to schedule servicing and repair events. Understanding the trajectory would also help evaluate the extent to which the observatory might continue to operate during the journey from the operating to the servicing location, or from the assembly to the operating location.

While the technology for electric propulsion from the assembly location (whether in LEO or L1) to deep-space is fairly well-developed, and demonstrated through recent missions such as Deep Space 1 and Genesis, the systems for transporting humans beyond LEO don't exist. It is assumed that the propulsion system will be high-energy chemical propulsion. The life support, communications, and structural systems will likely build on the technologies of the ISS and the Space Shuttle, while recognizing the differences between routine LEO operations and more distant destinations, involving higher radiation, longer communications lag times, and potentially longer stay times.

\section{REFERENCES}

[1] http://sohowww.nascom.nasa.govI

[2] http://www.srl.caltech.edu/ACE/

[3] http://map.gsfc.nasa.gov/

[4] W. Koon, M. Lo, J. Marsden, \& S. Ross, "Dynamical Systems, the Three-Body Problem and Space Mission Design", International Conference on Differential Equations, Berlin, 1999. p. 1176 in World Scientific, 2000.

[5] W. Koon, M. Lo, J. Marsden, \& S. Ross, "Shoot the Moon", American Astronautical Society AAS00-166.

[6] M. Lo \& S. Ross, "The Lunar Li Gateway: Portal to the Stars and Beyond", American Institute of Aeronautics and Astronautics, AIAA 2001-4768, Albuquerque, 2001.

[7] Rothenberg speech in American Institute of Aeronautics and Astronautics, Albuquerque, 2001.

[8] National Aeronautics and Space Administration, Strategic Plan 2000, Washington, DC, 2000.

[9] National Aeronautics and Space Administration, the space science enterprise strategic plan, Washington, DC, November 2000.

[10] "Orbital Express Space Operations Architecture / ASTRO" in http://www.darpa.mil/tto/programs/astro.html

[11] R. Ambrose, C. Culbert, \& F. Rehnmark, "An Experimental Investigation of Dexterous Robots Using EVA Tools and Interfaces", American Institute of Aeronautics and Astronautics, AIAA 2001-4593, Albuquerque, 2001.

[12] Gardell Gefke. Ranger Telerobotic Shuttle Experiment. http://rtsx.ssl.umd.edu/, May 2001. UMD SSL. 